\title{
Toward Circumventing Collinearity Effect in Nonlinear Spectral Mixture Analysis by Using a Spectral Shape Measure
}

\author{
Wei Yang, Akihiko Kondoh \\ Center for Environmental Remote Sensing, Chiba University, Chiba, Japan \\ Email: yangwei@chiba-u.jp
}

Received 29 June 2016; accepted 12 August 2016; published 15 August 2016

Copyright @ 2016 by authors and Scientific Research Publishing Inc.

This work is licensed under the Creative Commons Attribution International License (CC BY). http://creativecommons.org/licenses/by/4.0/

(c) $\underset{\mathrm{EY}}{0}$ Open Access

\begin{abstract}
Nonlinear spectral mixture analysis (NSMA) is a widely used unmixing algorithm. It can fit the mixed spectra adequately, but collinearity effect among true and virtual endmembers will decrease the retrieval accuracies of endmember fractions. Use of linear spectral mixture analysis (LSMA) can effectively reduce the degree of collinearity in the NSMA. However, the inadequate modeling of mixed spectra in the LSMA will also yield retrieval errors, especially for the cases where the multiple scattering is not ignorable. In this study, a generalized spectral unmixing scheme based on a spectral shape measure, i.e. spectral information divergence (SID), was applied to overcome the limitations of the conventional NSMA and LSMA. Two simulation experiments were undertaken to test the performances of the SID, LSMA and NSMA in the mixture cases of treesoil, tree-concrete and tree-grass. Results demonstrated that the SID yielded higher accuracies than the LSMA for almost all the mixture cases in this study. On the other hand, performances of the SID method were comparable with the NSMA for the tree-soil and tree-grass mixture cases, but significantly better than the NSMA for the tree-concrete mixture case. All the results indicate that the SID method is fairly effective to circumvent collinearity effect within the NSMA, and compensate the inadequate modeling of mixed spectra within the LSMA.
\end{abstract}

\section{Keywords}

Nonlinear Spectral Mixture Analysis, Linear Spectral Mixture Analysis, Collinearity, Spectral Information Divergence (SID)

\section{Introduction}

Land surfaces are inherently heterogeneous at large scales, and mixed pixels exist widely in remotely sensed 
images due to coarse spatial resolutions. The spectral mixture not only results in errors for the materials' discrimination and classification, but also greatly hinders the development of quantitative remote sensing. On the other hand, understanding abundances (fractions) of components (endmembers) will greatly benefit the modeling of biogeochemical cycles and climate at both global and regional scales [1] [2]. Therefore, it stimulates the development of spectral mixture analysis (SMA) techniques to quantify the fractions of the endmembers present in the mixed pixels.

The simplest SMA method is to model a mixed spectrum as a linear combination of the pure spectra of the endmembers weighted by their fractional coverage (i.e. Linear SMA, LSMA) [3]. This method is based on the hypothesis that incident solar radiation is reflected from earth surface through a single bounce and does not undergo multiple scattering within a pixel. Once appropriate spectra of endmembers have been determined, subpixel mapping can be performed using a fully constrained least squares method to impose the abundance sum-to-one constraint and abundance nonnegative constraint [4]. Problem is that when the endmembers are not arranged in discrete segregated patches on the surface, high intensity of multiple scattering usually happens [5]. For example, the interactions between soil and vegetation make the mixed spectra violate the linearity hypothesis [5]-[7].

Accordingly, a number of Nonlinear SMA (NSMA) methods have been developed [8]-[10], in which the multiple photo interactions have been taken into consideration. One typical NSMA method was developed based on physical principle of multiple scattering [5], and further adapted by [11]. Virtual endmembers (interaction terms) are introduced into the linear spectral model to reflect the multiple photo interactions in this method. It is denoted as NSMA for brevity hereafter in this study. The NSMA has been successfully applied to hyperspectral data in recent studies [6] [12]-[15]. Although the NSMA can fit the mixed pixel better than the LSMA, it does not guarantee that the endmember fractions can be retrieved with higher accuracy. This is because the degree of collinearity will be largely increased by introducing virtual endmembers in the NSMA, and consequently makes the retrieval errors increase dramatically with the increasing level of spectral noises [16] [17]. The spectral noises are inevitable in remotely sensed data, and very difficult to be quantified. A direct way to avoid collinearity is to exclude collinear variables (i.e. virtual endmembers in NSMA) [18], that is to use the LSMA. However, the LSMA cannot model the mixed spectra adequately when multiple scattering is not ignorable, and consequently yields estimation errors for endmember fractions.

To overcome the dilemmas for applying LSMA and NSMA to spectral mixture cases with significant multiple scattering, we applied a generalized spectral unmixing scheme proposed by [19] with a spectral-shape-based measure, i.e. spectral information divergence (SID). Two simulation experiments were undertaken to test the performance of the SID method. One is designed to test the sensitivity of the method to spectral noises, and the other is to test the sensitivity to interactive intensities among endmembers.

\section{Materials and Methods}

\subsection{LSMA and NSMA}

1) LSMA: The LSMA is reasonable when multiple photon interactions are believed to be negligible, in which the reflectance of a pixel is modeled as a weighted sum of the reflectance of each endmember within a pixel:

$$
\boldsymbol{R}=\sum_{i=1}^{M} f_{i} \boldsymbol{X}_{i}+\boldsymbol{e}
$$

where $\boldsymbol{R}=\left(R_{1}, R_{2}, \cdots, R_{N}\right)^{\mathrm{T}}$ is the mixed spectra; $\boldsymbol{X}_{i}=\left(X_{1, i}, X_{2, i}, \cdots, X_{N, i}\right)^{\mathrm{T}}$ is the reflectance spectra of endmember $i$; $f_{i}$ is the fraction of the endmember, $M$ is the number of endmembers, and $\boldsymbol{e}=\left(e_{1}, e_{2}, \cdots, e_{N}\right)^{\mathrm{T}}$ is the residual errors. The fractions of the endmembers are commonly constrained by

$$
\sum_{i=1}^{M} f_{i}=1 \text { and } 0 \leq f_{i} \leq 1
$$

Model fit is usually assessed by the root-mean-square-error (RMSE):

$$
\text { RMSE }=\sqrt{\frac{\sum_{k=1}^{N} e_{k}^{2}}{N}}
$$


2) NSMA: The NSMA accounts for the multiple photon interactions by introducing virtual endmembers into the LSMA. The virtual endmembers are presented by the cross-products of the true endmembers [6] [11]. The mixed spectrum is consequently expressed as following:

$$
\boldsymbol{R}=\sum_{i=1}^{M} c_{i} \boldsymbol{X}_{i}+\sum_{i=1}^{M} \sum_{j=1}^{M} c_{i, j} \boldsymbol{X}_{i, j}+\boldsymbol{e}, \text { with } c_{i} \geq 0, c_{i, j} \geq 0
$$

where $\boldsymbol{X}_{i, j}=\left(X_{1, i} X_{1, j}, X_{2, i} X_{2, j}, \cdots, X_{N, i} X_{N, j}\right)^{\mathrm{T}}$ denotes the interaction effect between the endmember $i$ and $j$; $c_{i}$, and $c_{i, j}$ are the contribution factors of the true and virtual endmembers. Reference [13] pointed out that the virtual endmember cannot be interpreted as an actual physical endmember of the ground cover, but they should satisfy the sum-to-one constraint from energy view. Therefore, normalization is conducted for contribution factors to satisfy this constraint, i.e.,

$$
\begin{gathered}
\sum_{i=1}^{M} c_{i}+\sum_{i=1}^{M} \sum_{j=1}^{M} c_{i, j}=K \\
f_{i}^{(1)}=\frac{c_{i}}{K}, \quad f_{i, j}^{(2)}=\frac{c_{i, j}}{K}
\end{gathered}
$$

where $f_{i}^{(1)}$ and $f_{i, j}^{(2)}$ are fractions of the true and virtual endmembers, respectively. According to the assumption that the probability of an endmember's interaction is proportional to its fraction in the pixel, coverage proportion of the endmember $i$ can be obtained as follows [12]:

$$
f_{i}=\frac{f_{i}^{(1)}}{1-\sum_{j=1}^{M} f_{i, j}^{(2)}}
$$

The model fit is also assessed by RMSE calculated as Equation (3).

\subsection{Disadvantage of the NSMA}

In the mixture cases that the three dimensional (3-D) objects are dominant, the NSMA can fit the mixed spectrum better than the LSMA with a smaller value of RMSE [6]. However, it does not guarantee that higher accuracies for the retrieval of endmembers' fractions can be derived. This is mainly because the introduced interaction terms may largely increase the degree of collinearity among endmembers in the NSMA [16], especially when spectra of some endmembers are relatively flat. Collinearity here means that there is a high correlation or dependence between the endmembers, resulting in some endmembers being explained by the others. The high degree of collinearity may make the retrieval of endmember fractions change erratically in response to minor changes in noise level in the matrix deconvolution process, and consequently reduce the inversion accuracy.

\subsection{Spectral-Shape-Based Unmixing Method}

To circumvent the collinearity effect resulted from the interaction terms, a generalized unmixing framework was utilized, in which spectrum of a target pixel was matched with a modeled spectrum, and the matching criterion can be either spectral-shape-based or spectral-magnitude-based measures [19]. Mathematically, it is described as following:

$$
\begin{array}{r}
\min d\left(\boldsymbol{R}, \boldsymbol{R}_{\text {mod }}\right)=G\left(f_{1}, f_{2}, \cdots, f_{M}\right) \\
\text { s.t. } \sum_{i=1}^{M} f_{i}=1 \text { and } 0 \leq f_{i} \leq 1
\end{array}
$$

where $\boldsymbol{R}$ is the target spectrum; $\boldsymbol{R}_{\text {mod }}$ is the spectrum modeled as the weighted linear sum of the endmembers' spectra (same as the LSMA); $G$ is the objective function, which is the spectral matching criterion selected to measure the difference between $\boldsymbol{R}$ and $\boldsymbol{R}_{\bmod }$; the fractions of the endmembers, $f_{1}, f_{2}, \cdots, f_{M}$, are the decision variables. The constraints are the same as Equation (2). Equation (8) represents a constrained nonlinear optimization problem, which can be solved using the sequential quadratic programming [19]. Advantage of this me- 
thod is that any spectral similarity measure can be accommodated.

To compensate the modeling errors caused by multiple scattering, the spectral information divergence (SID), which is a spectral-shape-based measure, is used in this study because of its high effectiveness [19] [20]. The SID is a measure of the discrepancy between two spectra based on their corresponding probability mass functions, and the corresponding objective function $G$ is defined as:

$$
G_{\mathrm{SID}}=\sum_{i=1}^{N} p_{i} \log \left(\frac{p_{i}}{q_{i}}\right)+\sum_{i=1}^{N} q_{i} \log \left(\frac{q_{i}}{p_{i}}\right)
$$

where $p_{i}$ and $q_{i}, \quad i=1,2, \cdots, N$, the two probability mass functions produced by the modeled spectrum and target spectrum, respectively, are defined as follows:

$$
p_{i}=\frac{R_{\mathrm{mod}, i}}{\sum_{i=1}^{N} R_{\mathrm{mod}, i}}, \quad q_{i}=\frac{R_{i}}{\sum_{i=1}^{N} R_{i}}
$$

\subsection{Collection of Endmembers' Spectra}

In order to carry out the simulation analysis for evaluating the performances of the unmimxing methods, reflectance spectra of endmembers were collected from the spectral library contained in the software ENVI. Specifically, reflectance spectra of tree, soil, concrete and grass were derived from the John Hopkins University spectral library. All the spectra data sets were resampled to the spectral range of $400-2400 \mathrm{~nm}$ with an interval of 1 $\mathrm{nm}$. Figure 1 shows the mixture groups of tree-soil, tree-concrete and tree-grass, as well as their products (i.e. the visual endmembers in the nonlinear mixture model).

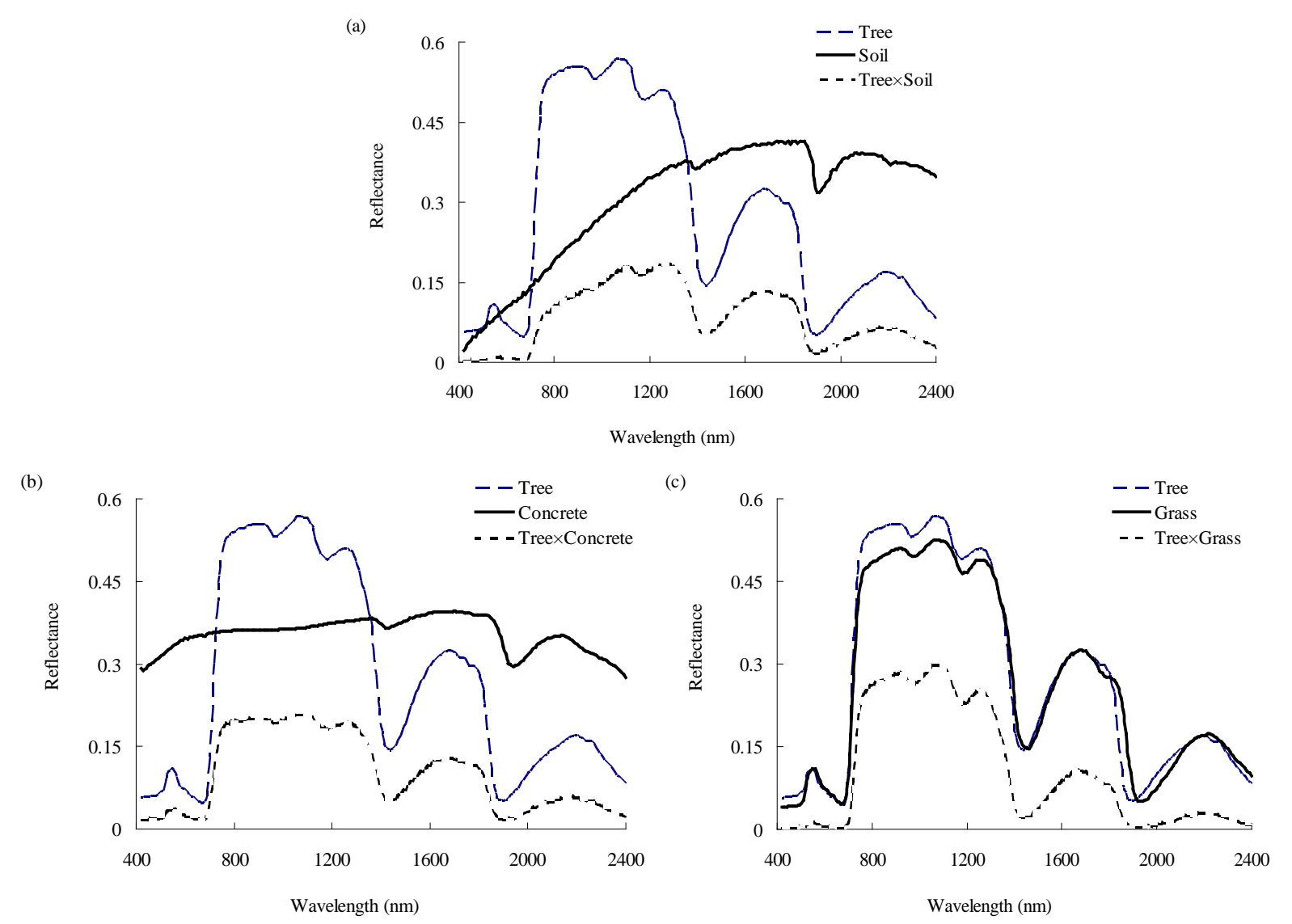

Figure 1. Reflectance spectra of endmembers for simulation experiments with different mixture cases: (a) tree-soil mixture; (b) tree-concrete mixture; (c) tree-grass mixture. 


\subsection{Generation of Mixed Spectra}

The spectra were grouped as tree-soil mixture, tree-concrete mixture and tree-grass mixture as shown in Figures 1(a)-(c). Mixed pixels were generated according to the method in [12]:

$$
\begin{gathered}
\qquad \boldsymbol{R}=c_{1} \boldsymbol{X}_{1}+c_{2} \boldsymbol{X}_{2}+c_{1,2} \boldsymbol{X}_{1} \boldsymbol{X}_{2}+\boldsymbol{\sigma} \\
\text { with } c_{1}=f_{1}\left(1-c_{12}\right), \quad c_{2}=f_{2}\left(1-c_{12}\right)
\end{gathered}
$$

For each two-endmember combination, two groups of mixed spectra were synthesized with different levels of Gaussian noise $(\sigma)$ and nonlinear mixing intensities (i.e. $\left.c_{12}\right)$.

The Group I data were generated to test the sensitivity of the unmixing methods to the Gaussian noise contained in the spectra, in which $c_{12}$ was set as a constant value of 0.15 for each spectrum. Values of $f_{1}$ increased from $0 \%$ to $100 \%$ with steps of $1 \%$, and correspondingly $f_{2}=1.0-f_{1}$. Consequently 101 mixed spectra were derived through Equation (11). Then the Gaussian white noises were added into the synthesized spectra. The standard deviation $(\sigma)$ of Gaussian white noise changed from 0 to 0.1 with steps of 0.01 . Corresponding to each $\sigma$, random numbers used to imitate Gaussian noise were run 500 times.

The Group II data were generated to test the sensitivity of the unmixing methods to the nonlinear mixing intensities. Values of $f_{1}$ and $f_{2}$ were determined as in the first dataset, while the $c_{12}$ was changed from 0 to 0.2 with steps of 0.02. Gaussian white noises were added with the constant standard deviation of 0.05 . Corresponding to each $c_{12}$, the Gaussian noises were also run 500 times with different random numbers.

Performance of the unmixing method was assessed by comparing the estimated and true tree fractions. For the first dataset, RMSE corresponding to each level of $\sigma$ was calculated as follows:

$$
\text { RMSE }=\sqrt{\frac{\sum_{k=1}^{101}\left(f_{\text {tree }, k}-f_{\text {esti, } k}\right)^{2}}{101}}
$$

in which $f_{\text {tree, } k}$ and $f_{\text {esti, } k}$ are the true and estimated tree fraction. There would be 500 values of RMSE corresponding to 500 runs of Gaussian noises for each $\sigma$. Consequently, the mean and standard deviation (SD) of these RMSEs were calculated. The same calculation was performed in the second dataset for each level of nonlinear mixing intensities (i.e. $c_{12}$ ).

\section{Results}

\subsection{Collinearity among Endmembers in the LSMA and NSMA}

The degree of collinearity among the endmembers in spectral mixture models can be quantified by using the so-called Variance Inflation Factor (VIF, [18]). The higher value of the VIF is, the higher degree of collinearity there is. Figure 2 shows the VIF in the LSMA and the average VIF in the NSMA for different mixture cases. It can be seen that for the case of tree-soil mixture, the NSMA has a larger VIF than the LSMA (8.9 vs. 1.3), while the degrees of colinearity are very low for both the LSMA and NSMA. For the case of tree-concrete mixture, which would be encountered in urban areas, the VIF of NSMA is about one hundred and twenty times larger than that of the LSMA (167.0 vs. 1.4), showing that the degree of collinearity is dramatically increased by the NSMA. It is because that the reflectance spectrum of concrete is relatively flat, and then the interaction term (tree $\times$ concrete) is highly correlated with tree spectrum (Figure 1(b)). For the case of tree-grass mixture, both the LSMA and NSMA have large values of VIFs since the high correlation between the spectra of tree and grass, while the NSMA has a slightly larger one (Figure 1(c)). The results indicate that the degree of collinearity will be increased by the NSMA, while the amount of increase depends on the spectral shape of endmembers.

\subsection{Simulation Experiment (I): Unmixing Performance vs. Gaussian Noise}

The SID method was first applied to the Group I simulation dataset. For comparison, the conventional LSMA and NSMA as demonstrated in [12] have also been implemented for the same dataset. Performances of the unmixing methods were evaluated by RMSE (i.e. Equation (12)) for the estimation of tree fraction.

Figures 3(a)-(c) show the mean RMSE of tree-fraction estimation against the standard deviation of Gaussian noises $(\sigma)$ for mixture cases of tree-soil, tree-concrete, and tree-grass, respectively. The error bars denote standard 


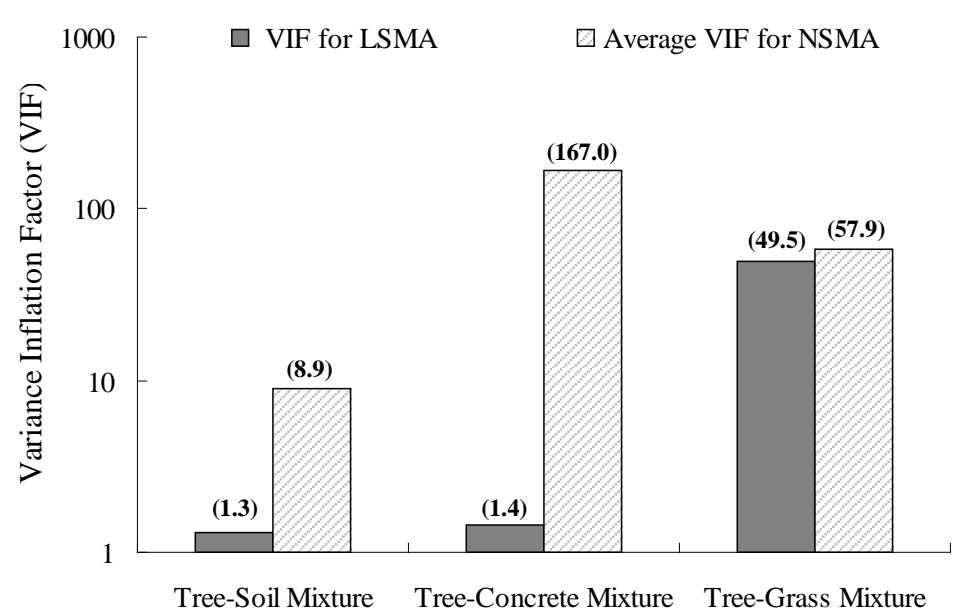

Figure 2. Variation Inflation Factor (VIF) values of endmembers in Linear Spectral Mixture Analysis (LSMA) and Nonlinear Spectral Mixture Analysis (NSMA) for the simulation datasets.
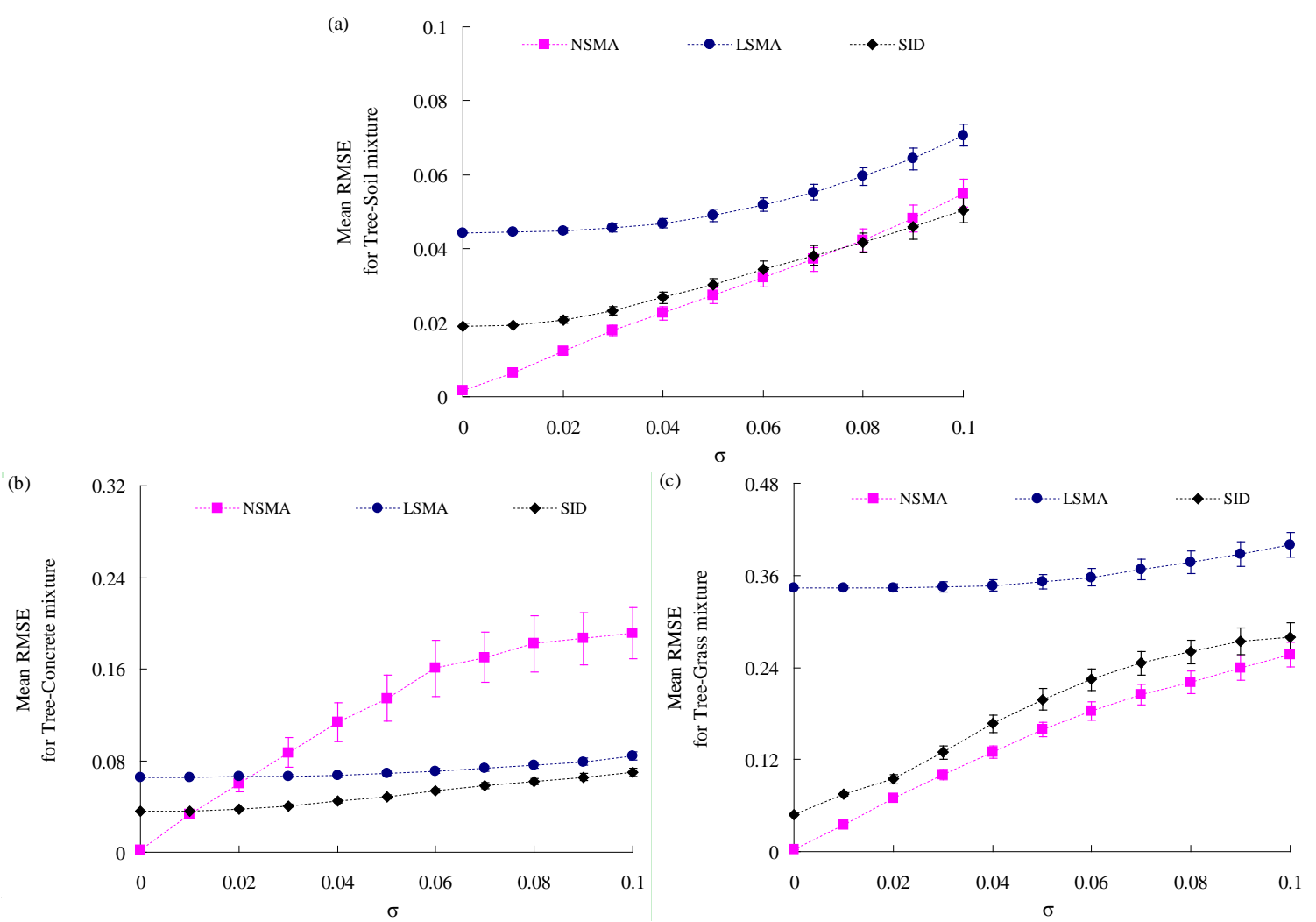

Figure 3. Relationship between RMSEs and the amount of Gaussian noise $(\sigma)$ (with $c_{12}=0.15$ ) for (a) tree-soil mixture; (b) tree-concrete mixture; and (c) tree-grass mixture.

deviation for the 500 RMSEs corresponding to each level of $\sigma$. It was found that the SID method outperformed the conventional LSMA significantly for all mixture cases. This is because the conventional LSMA is implemented using brightness-based measure, and then the spectral mixture modeling errors will largely affect the retrieval accuracies. In contrast, the SID method applying the spectral-shape-based measure can compensate the modeling errors to some extent. Compared with the conventional NSMA, the SID method yielded similarly small RMSE $(<0.05)$ for the tree-soil mixture, except that when the $\sigma$ is quite small, the RMSE for NSMA is relatively lower (Figure 3(a)). While for the tree-concrete mixture, estimation errors of the conventional NSMA 
dramatically increased with the increasing $\sigma$ (Figure 3(b)). In contrast, the SID method showed relatively stable pattern with the increase of $\sigma$. When $\sigma$ was low, the conventional NSMA yielded slight higher accuracy than the SID method; while the $\sigma$ was beyond 0.01 the RMSEs of SID was noticeably lower than the conventional NSMA. Large variability of estimation accuracy for the NSMA was also observed with wide error-bars when $\sigma$ was greater than 0.04. For the tree-grass mixture, RMSEs of the SID and conventional NSMA were both proportional to the $\sigma$ (Figure 3(c)), due to the high degree of collinearity among the endmembers.

\subsection{Simulation Experiment (II): Unmixing Performance vs. Interactive Intensity}

The SID method, as well as conventional LSMA and NSMA were then applied to the Group II simulation dataset, in which the parameter $c_{12}$ denotes the interactive intensity in the spectral mixture model. Figure 4 shows the unmixing errors against the levels of interactive intensity $\left(c_{12}\right)$ when the level of Gaussian noise is fixed ( $\sigma=$ 0.05).

Results showed that RMSEs of the conventional LSMA increased rapidly when the $c_{12}$ increased in all mixture cases, while the SID method showed relatively stable patterns against the increasing $c_{12}$ (Figures 4(a)-(c)). The RMSEs of the SID were slightly higher than the conventional LSMA when $c_{12}$ was low; when the $c_{12}$ was larger than a certain value (e.g., about 0.04 for the tree-soil mixture), the SID method yielded noticeably higher accuracy than the conventional LSMA. Compared with the conventional NSMA, the SID method yielded similar accuracies for the mixture cases of tree-soil and tree-grass. While for the tree-concrete mixture, the SID method significantly outperformed the conventional NSMA, totally because of the dramatically increased degree of collinearity among the endmembers in the NSMA.

\section{Discussion}

Advantages of the spectral-shape-based unmixing method, SID, are mainly in two aspects. First, the modeling
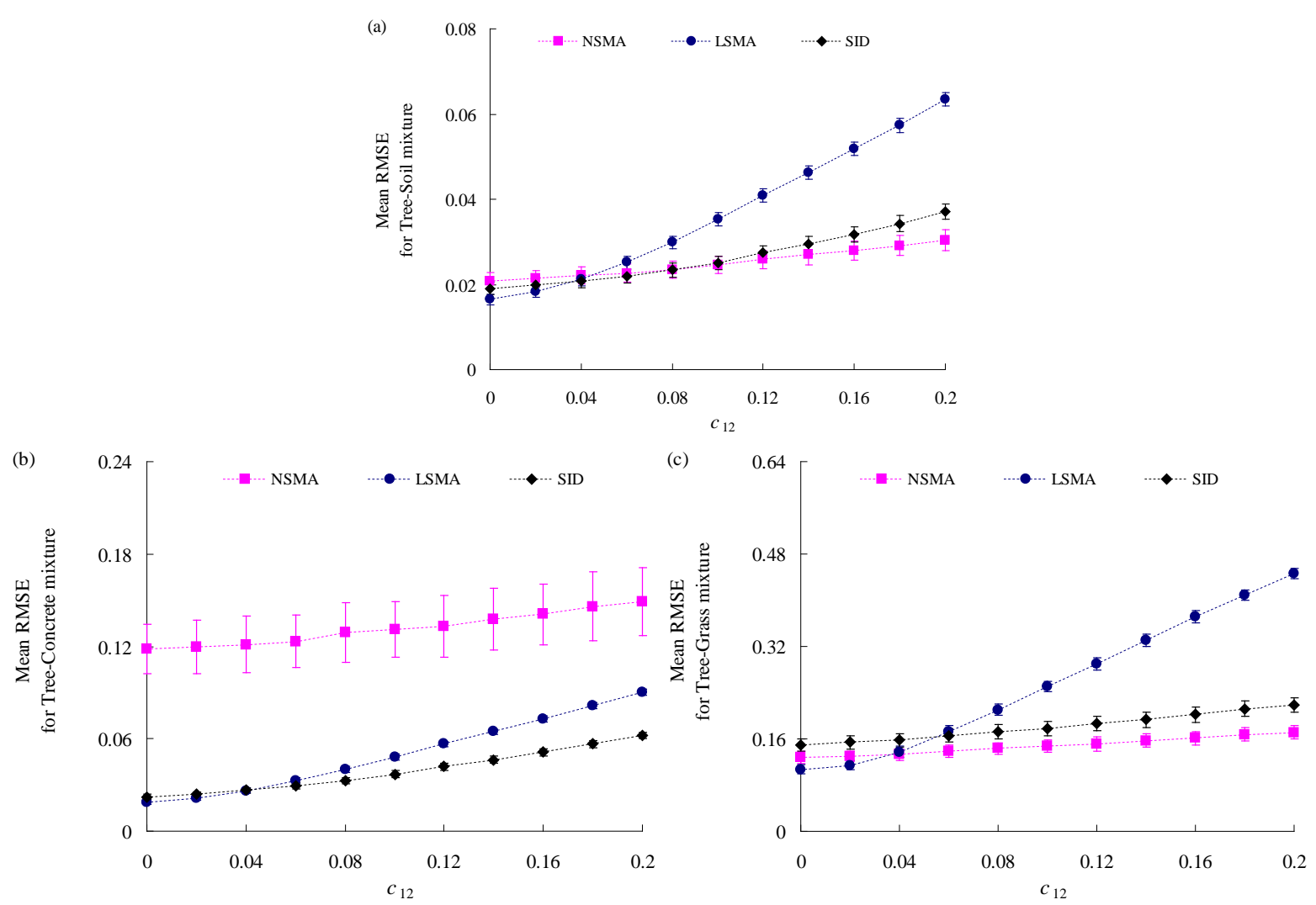

Figure 4. Relationship between RMSEs and the amount of nonlinear interaction intensities ( $c_{12}$ ) (with $\sigma=0.05$ ) for (a) tree-soil mixture; (b) tree-concrete mixture; and (c) tree-grass mixture. 
errors results from the linear mixture model can be compensated by using the spectral-shape-based measure as the matching criterion. This explains the better performance of the SID than the conventional LSMA when the interactive intensities are relatively high. Second, the increased degree of collinearity due to the interaction terms in the NSMA can be simply circumvented by using the linear mixture model. The merit of using the linear model was apparently demonstrated in the case of tree-concrete mixture, in which the SID significantly outperformed the conventional NSMA. It should be noted that the SID cannot overcome the collinearity between true endmembers (e.g., tree and grass), while its accuracy is comparable with the NSMA even for that case.

Another potential merit of the SID method is that the spectral-shape-based measure is less sensitive to magnitude variations of the mixed spectra. Therefore, it has lower requirement for exact atmospheric correction than the conventional brightness-based algorithms, which can avoid the difficulties in obtaining all the atmospheric parameters [19].

The simulation experiments in this study are designed to imitate the simplest two-endmember mixtures with 3-D structures (i.e. the tree), showing three typical variations of collinearity effect between the LSMA and NSMA. These are: 1) the collinearity of LSMA and NSMA are both low, i.e. tree-soil mixture; 2) the LSMA is low, but in contrast the NSMA is very high, i.e. tree-concrete mixture; and 3) the LSMA and NSMA are both high, i.e. tree-grass mixture. Natural circumstances would be much more complicated than the simulated cases. However, it can be deduced that more complicated experiments will not change the characteristics of collinearity for the LSMA and NSMA, and consequently will not yield different results from this study. Additionally, application of the SID in more field measurements and/or satellite images will be investigated as future works.

\section{Conclusion}

In this study, the SID was applied to circumvent the collinearity effect in nonlinear mixture model. Simulation data sets with different levels of Gaussian noises and nonlinear mixing intensities were generated to test the performances of the SID in mixture cases of tree-soil, tree-grass, and tree-concrete. Results demonstrated that the SID method is generally more flexible than the conventional LSMA and NSMA. The SID outperforms the conventional LSMA for almost all the mixture cases, and performs significantly better than the NSMA for the tree-concrete mixture. For the mixture cases of tree-soil and tree-grass, the performance of the SID method is comparable with the NSMA. The results indicate the potential of SID method in circumventing the collinearity effect in NSMA caused by the virtual endmembers. In future works, the SID method will be applied to field measurements and satellite images to make further validations.

\section{Acknowledgements}

This research was conducted under the JAXA GCOM Research Announcement (the 4th RA 102 and the 6th RA 111).

\section{References}

[1] Sellers, P.J., et al. (1997) The Impact of Using Area-Averaged Land Surface Properties-Topography, Vegetation Condition, Soil Wetness-In Calculations of Intermediate Scale (Approximately $10 \mathrm{~km}^{2}$ ) Surface-Atmosphere Heat and Moisture Fluxes .Journal of Hydrology, 190, 269-301. http://dx.doi.org/10.1016/S0022-1694(96)03130-7

[2] Mayes, M.T., Mustard, J.F. and Melillo, J.M. (2015) Forest Cover Change in Miombo Woodlands: Modeling Land Cover of African Dry Tropical Forests with Linear Spectral Mixture Analysis. Remote Sensing of Environment, 165, 203-215. http://dx.doi.org/10.1016/j.rse.2015.05.006

[3] Adams, J.B., Smith, M.O. and Johnson, P.E. (1986) Spectral Mixture Modeling: A New Analysis of Rock and Soil Types at the Viking Lander I Site. .Journal of Geophysical Research, 91, 8098-8112. http://dx.doi.org/10.1029/JB091iB08p08098

[4] Heinz, D.C. and Chang, C.I. (2001) Fully Constrained Least Squares Linear Spectral Mixture Analysis Method for Material Quantification in Hyperspectral Imagery. IEEE Transactions on Geosciences and Remote Sensing, 39, 529545. http://dx.doi.org/10.1109/36.911111

[5] Borel, C.C. and Gerstl, S.A.W. (1994) Nonlinear Spectral Mixture Models for Vegetative and Soil Surfaces. Remote Sensing of Environment, 47, 403-416. http://dx.doi.org/10.1016/0034-4257(94)90107-4

[6] Chen, X.X. and Vierling, L. (2006) Spectral Mixture Analyses of Hyperspectral Data Acquired Using a Tethered Balloon. Remote Sensing of Environment, 103, 338-350. http://dx.doi.org/10.1016/j.rse.2005.05.023 
[7] Ma, L., et al. (2015) Estimation of Fractional Vegetation Cover in Semiarid Areas by Integrating Endmember Reflectance Purification into Nonlinear Spectral Mixture Analysis. IEEE Geoscience and Remote Sensing Letters, 12, 11751179 http://dx.doi.org/10.1109/LGRS.2014.2385816

[8] Carpenter, G.A., et al. (1999) A Neural Network Method for Mixture Estimation for Vegetation Mapping. Remote Sensing of Environment, 70, 138-152. http://dx.doi.org/10.1016/S0034-4257(99)00027-9

[9] DeFries, R.S., Townshend, J.R.G. and Hansen, M.C. (1999) Continuous Fields of Vegetation Characteristics at the Global Scale at 1-km Resolution. Journal of Geophysical Research-Atmospheres, 104, 16911-16923. http://dx.doi.org/10.1029/1999JD900057

[10] Ju, J.C., Kolaczyk, E.D. and Gopal, S. (2003) Gaussian Mixture Discriminant Analysis and Sub-Pixel Land Cover Characterization in Remote Sensing. Remote Sensing of Environment, 84, 550-560. http://dx.doi.org/10.1016/S0034-4257(02)00172-4

[11] Ray, T.W. and Murray, B.C. (1996) Nonlinear Spectral Mixing in Desert Vegetation. Remote Sensing of Environment, 55, 59-64. http://dx.doi.org/10.1016/0034-4257(95)00171-9

[12] Somers, B., et al. (2009) Nonlinear Hyperspectral Mixture Analysis for Tree Cover Estimates in Orchards. Remote Sensing of Environment, 113, 1183-1193. http://dx.doi.org/10.1016/j.rse.2009.02.003

[13] Zhang, L., et al. (1998) Study of the Spectral Mixture Model of Soil and Vegetation in PoYang Lake Area, China. International Journal of Remote Sensing, 19, 2077-2084. http://dx.doi.org/10.1080/014311698214875

[14] Arai, K. (2007) Nonlinear Mixture Model of Mixed Pixels in Remote Sensing Satellite Images Based on Monte Carlo Simulation. Advances in Space Research, 41, 1715-1723. http://dx.doi.org/10.1016/j.asr.2007.04.096

[15] Tong, X., et al. (2016) Development of In Situ Experiments for Evaluation of Anisotropic Reflectance Effect on Spectral Mixture Analysis for Vegetation Cover. IEEE Geoscience and Remote Sensing Letters, 13, 636-640. http://dx.doi.org/10.1109/LGRS.2016.2531743

[16] Chen, X.H., et al. (2011) A Quantitative Analysis of Virtual Endmembers' Increased Impact on the Collinearity Effect in Spectral Unmxing. IEEE Transactions on Geosciences and Remote Sensing, 49, 2945-2956. http://dx.doi.org/10.1109/TGRS.2011.2121073

[17] Ma, L., et al. (2016) Two-Step Constrained Nonlinear Spectral Mixture Analysis Method for Mitigating the Collinearity Effect. IEEE Transactions on Geoscience and Remote Sensing, 54, 2873-2886. http://dx.doi.org/10.1109/TGRS.2015.2506725

[18] Weisberg, S. (1985) Applied Linear Regression. Wiley, New York.

[19] Chen, J., et al. (2009) Generalization of Subpixel Analysis for Hyperspectral Data with Flexibility in Spectral Similarity Measures. IEEE Transactions on Geosciences and Remote Sensing, 47, 2165-2171. http://dx.doi.org/10.1109/TGRS.2008.2011432

[20] Van der Meer, F. (2006) The Effectiveness of Spectral Similarity Measures for the Analysis of Hyperspectral Imagery. International Journal of Applied Earth Observation and Geoinformation, 8, 3-17. http://dx.doi.org/10.1016/j.jag.2005.06.001

\section{Submit or recommend next manuscript to SCIRP and we will provide best service for you:}

Accepting pre-submission inquiries through Email, Facebook, LinkedIn, Twitter, etc.

A wide selection of journals (inclusive of 9 subjects, more than 200 journals)

Providing 24-hour high-quality service

User-friendly online submission system

Fair and swift peer-review system

Efficient typesetting and proofreading procedure

Display of the result of downloads and visits, as well as the number of cited articles

Maximum dissemination of your research work

Submit your manuscript at: http://papersubmission.scirp.org/ 